\title{
The Shock Wave in the ionosphere during an Earthquake
}

\author{
Vladimir Kuznetsov \\ Institute of cosmophysical research and radio wave propagation FEB RAS 684034 Kamchatka, Russia
}

\begin{abstract}
Fundamentally new model of the shock wave (SW) generation in atmosphere and ionosphere during earthquake is proposed. The model proceeds from the idea of cooperative shock water crystallization in a cloud.
\end{abstract}

\section{Introduction}

Fundamentally new shock-wave model of an earthquake has being developed by the author during the last 25 years. Pr. Afraimovitch E.L. whom I presented my model in 2004 in IKIR approved it and acquainted me with his papers [2] on the shock acoustic waves (SAW) in atmosphere detected through the analysis of total electrons content (TEC) signals recorded by GPS-receivers (Fig.1).

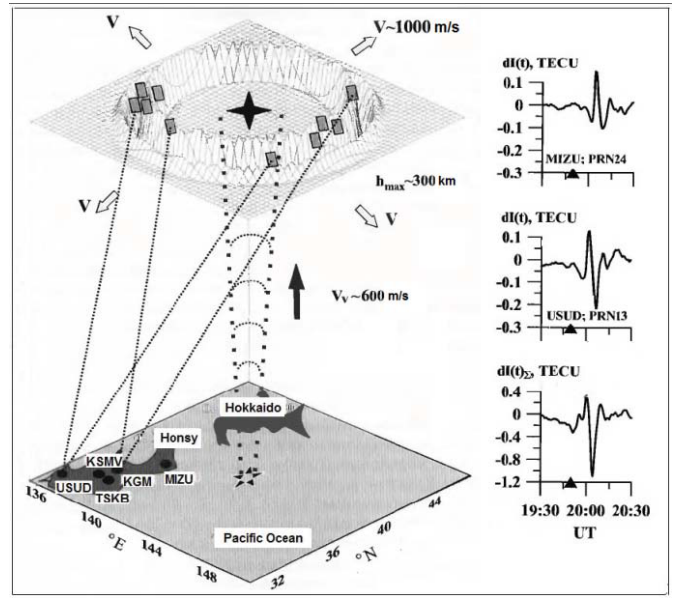

Fig. 1. Scheme of generation and detecting of SAW during the earthquake in Japan, September 25, 2003. Asterisk - earthquake epicenter, cross - secondary source, points - GPS-sensors, rectangles - ionospheric points. Right - TEC-variation during the earthquake near Hokkaido, September 25, 2003 [2].

Seeing the details of their research I didn't accept Pr. Afraimovitch' team interpretation of the phenomenon of SAW generation in ionosphere. What is known about the mechanism of ionospheric disturbances generation caused by seismic activity? As stated in [2, P. 338], such mechanism "is not clear". Modern approach to solving this problem consists in substituting the earthquake region for its point source [2, 3, 4]. The point underground source generates spherical elastic waves in mountain rock. The wave generation at the earth surface is similar to the generation of a strong shock when rock sections lift one after another and eventually return to their starting positions. The process is accompanied by the acoustic wave generation. The width of direction plot for the acoustic signal propagating from the perturbed Earth surface is very narrow [4]. Therefore, an acoustic disturbance penetrates up to the ionosphere altitudes within the narrow range of zenith angles. Moreover, the part of ionosphere above the epicenter is the source of ionospheric response. This model of ionosphere disturbances is developed in [3].

There are numerous results of observations of earthquake-induced strong ground motions and shock wave generation in various media. The fact that during powerful earthquakes the velocities of vertical ground motions are much less than the sound velocity in air opposes to the model of SAW generation proposed in [3]. The strong ground motions recorded during the Northridge earthquake will be taken as an example. Nevertheless, the observations on behavior of the electron density waves in ionosphere (Fig.1) prove the existence of SAW. This problem requires the development of principally new approaches.

Unfortunately, I began my research too late (2014) and I could not report about my results to Eduard Leontievitch who died (2009). I devote my manuscript to the blessed memory of Pr. Afraimovitch E.L.

Corresponding author: vvkuz38@mail.ru 


\section{Strong ground motions recorded during the Northridge earthquake}

The Northridge earthquake (magnitude of 6.7, January 17, 1994) is one of the most studied. It occurred nearby Hollywood, the seismically dangerous and populous region of Los Angeles, with the large number of the seismic measuring equipment [5]. Data on three components of the strong ground motions obtained with the devices of Van Norman Complex [6] and the results on the motion magnitude and direction are especially essential.

The values of vertical and horizontal amplitudes and velocities of ground motions were obtained there. Amplitudes of horizontal motions were twice-three times more than vertical ones which were about $h \approx 10-20 \mathrm{~cm}$. The peak velocity of horizontal ground motion was about $177 \mathrm{~cm} / \mathrm{s}$, the vertical average velocity was $10-20 \mathrm{~cm} / \mathrm{s}$. The peak amplitude of horizontal motions was up to $40 \mathrm{~cm}$. The hypothetical wavelength of vertical motions was $\lambda \approx$ not more than $50 \mathrm{~cm}$. At the shock moment the ground has lifted on about $\approx 50 \mathrm{~cm}$.

\section{SAW record in ionosphere at the earthquake}

Pr. Afraimovitch' team evaluated the height $h$ of assumed source $(h \approx 300 \mathrm{~km})$. Not epicenter but the region of ionospheric layer at $F_{2}$ altitude over epicenter was the source of ionospheric disturbances. The secondary source initiates in 8-9 minutes after the main shock. The calculated velocity of signal propagation through atmosphere is about $600 \mathrm{~m} / \mathrm{s}$ which is nearly twice as large as the experimental value for the speed of sound. It is easy to show that this model is incorrect.

\section{Our model}

Discussed model tries to interpret the occurrence of quantum entanglement between lithospheric and atmospheric processes. Entanglement may be activated with hydrogen bonds protons available in water and in many other geologic media such as kalicinite.

\section{SW generation in lithosphere}

Let's discuss the experiments on high-pressured kalicinite (KHCO3), the mineral in which hydrogen bonds show the capacity for quantum entanglement. On compression the structure of kalicinite hydrogen bonds changes simultaneously over the whole volume of the sample (Fig. 2) resulting in the change of its unit-cell volume (or density) and correspondingly (according to our model) in the SW generation. Similar data are obtained for other minerals containing hydrogen and oxygen (nitrogen, fluorine). By data of many authors such structural phase transition of hydrogen bonds happens instantly over the large volume and this phenomenon is called a cooperativity.

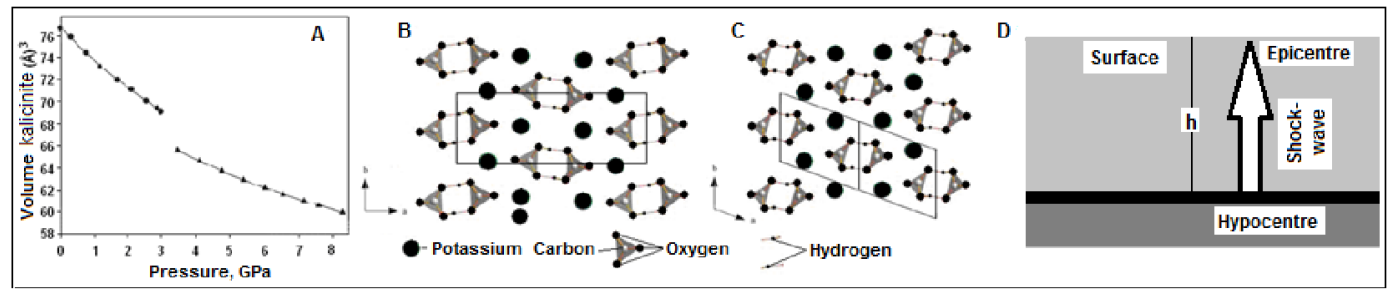

Fig. 2. The change of the unit-cell volume $(\Delta V=V=4 \%)$ for kalicinite at the pressure of $3.2 \mathrm{GPa}-A$, the change of kalicinite crystal structure - B,C. [7]. Shock wave generation inside the layer of lithosphere containing hydrogen bonds - D.

\section{Hydrogen bond}

A hydrogen bond occurs when the electron-lacked hydrogen covalently bound to the electronegative atom (oxygen, nitrogen, fluorine) interacts with lone-electron pair of another electronegative atom in the same molecule or in the other one. Cooperativity of hydrogen bonds in water in particular is responsible for the dependence of water features on the amount of hydrogen bonds. A sizeable quantity of hydrogen bonds in ice decreases with its melting up to their almost absence in boiling water. A lack of possibility to form hydrogen bonds would result in water transformation into vapor. Hydrogen bonding also plays an important role in lithosphere properties. Shifting of electron density at the line $\mathrm{H}$... O (N, F, etc.) to the electronegative atom provides hydrogen bonding which produces interatomic distances $\mathrm{O}-\mathrm{O}$, $\mathrm{O}-\mathrm{N}$ shorter than in hydrogen absence. 


\section{Earthquake energy}

We are going to estimate the energy needed for kalicinite (KHCO3) compression (see Fig 2-A). The external pressure $p=3 \mathrm{GPa}$ leads to a change in the sample volume $\Delta V / V \approx 5 \%$ that is about $10^{2} \mathrm{~J} / \mathrm{cm}^{3}$. In Fig. 2-B and C the socalled structural phase transition "order-disorder" is shown. In [7] the enthalpies of this transition are given for a wide range of crystals. We assume that energy of one broken hydrogen bond is about $0.5 \mathrm{eV}\left(1 \mathrm{eV} \approx 10^{-19} \mathrm{~J}\right)$. Let's consider how the energy released at fracture (fracture, rotation, etc.) of hydrogen bonds is consistent with the estimate obtained from the relation $E=p V$. In minerals under the external pressure the presence of hydrogen bonds can provide the energy necessary for the shock wave generation.

\section{Quantum entanglement}

Quantum entanglement is a perspective research direction included into the list of 20 Big Scienti_c Ideas in "BBC Focus Special Edition, 2013". Here we try to link such notions as 'entanglement', 'decoherence', 'recoherence' and 'teleportation' with crystallization and different types of phase transitions that can occur in lithosphere and atmosphere.

Since we do not exploit measurement, we can capture 'entanglement' through collective decoherence without common environment. In our earthquake model the generation of the shock wave follows from decoherence of multiparticle quantum system and subsequent fast phase transitions. Moreover, decoherence can transform one quantum system into another which can have additional properties revealed in the form of an aftershock, teleportation of proton state and ect.

\section{Quantum decoherence}

Decoherence (D) of quantum system (QS) occurs every time when its state becomes entangled with a state of the environment. Thus, 'information' of QS is imprinted in a state of its environment. It is known that D is a physical process accompanied with the loss of coherence because of entanglement with the environment. Moreover, after decoherence QS gets classical features reflected into environment. Under natural conditions it is impossible to isolate multiparticle QS. Therefore, open quantum systems are common. Because of entanglement with the environment a coherent state transforms into a 'classical' mixed one. Moreover, an inverse transition called recoherence is possible. For recoherence there is only need in weakening of interaction between system and environment. Since there is 'information' about quantum states imprinted in the environment, we can observe transformations of 'classical' environment induced by decoherence or recoherence. Due to the no-go theorem it is impossible to statistically verify these transformations. This idea prohibits prediction of an earthquake.

\section{Quantum teleportation}

A quantum teleportation (QT) is a transition of quantum state for arbitrary long distances with the help of classical channel and separation of quantum pairs. We can a priori assume that there are entangled QS separated by a distance. Since we cannot exclude the classical interaction (classical channel), we deal with QT. In some cases the classical channel is not obvious, for example, it is not clear how protons in hydrogen bonds of supercooled water in Arctic cyclones are classically linked with protons in hydrogen-bonded camphora of a storm-glass in Novosibirsk [8].

Another example of QT is a 'connection' between the Sumatra earthquake in 2004 and typical fluctuation of atmospheric electric field in Italy [9]. There are numerous examples of 'superluminal connection' between far distanced separated earthquakes. QT can be the only reason of such connections. It seems that sprites, jerks and particles of high energy in water saturated atmosphere are generated because of QT. By the way, here a lighting bolt is a 'classical channel'.

\section{Quantum nature of aftershocks}

The earthquake time series (aftershocks, also) is flicker-noise (see the Gutenberg-Richter law). In recent years the study of open quantum systems showed that flicker-noise accompanies decoherence. It follows that the nature of earthquakes, including aftershocks, is quantum. If the conditions in a QS allows full decoherence, the entire system is converted into a classical one with generating shock wave. If there is only a partial decoherence, then the second, third and etc. SW may occur after formation of the first shock wave. It follows that the formation of the aftershock differs.

\section{What is SAW?}

Interesting video clips of model tests of a shock-acoustic "gun" are available online. SAW generated by this device leads to the destruction of a specially constructed models of buildings, located at a distance of several dozen 
meters from the cannon. In the chamber of the gun SAW is generated under the bulk detonation of explosives in the form of aerosols. Nothing is discarded from the "barrel" of the gun during the shot, the SW which effects the destruction is only emitted. These experiments can be considered as the mechanistic model of the considered natural phenomenon of generation of SAW. In our case, the shock crystallization of supercooled water in the cloud plays the role of a SAW generator (detonator).

\section{Shock crystallization and generation of SAW}

Our model of SAW is alternative to the conventional one, shown in Fig.3. The model is based on experimental and theoretical studies of the shock crystallization in supercooled water. The model simultaneously solves two problems: the occurrence of hole-punch clouds and the existence of "thunder in a clear sky" phenomenon. The energy difference between the pieces of ice and drops with ice nuclei (Fig. 3-b) determines the energy of SAW. In the cloud of supercooled water there are conditions for a shock crystallization (Fig. 3-a). QS of a cloud can be quantum entangled with hydrogen bonds in the lithosphere. Here SW in a cloud is an aftershock of the main earthquake.
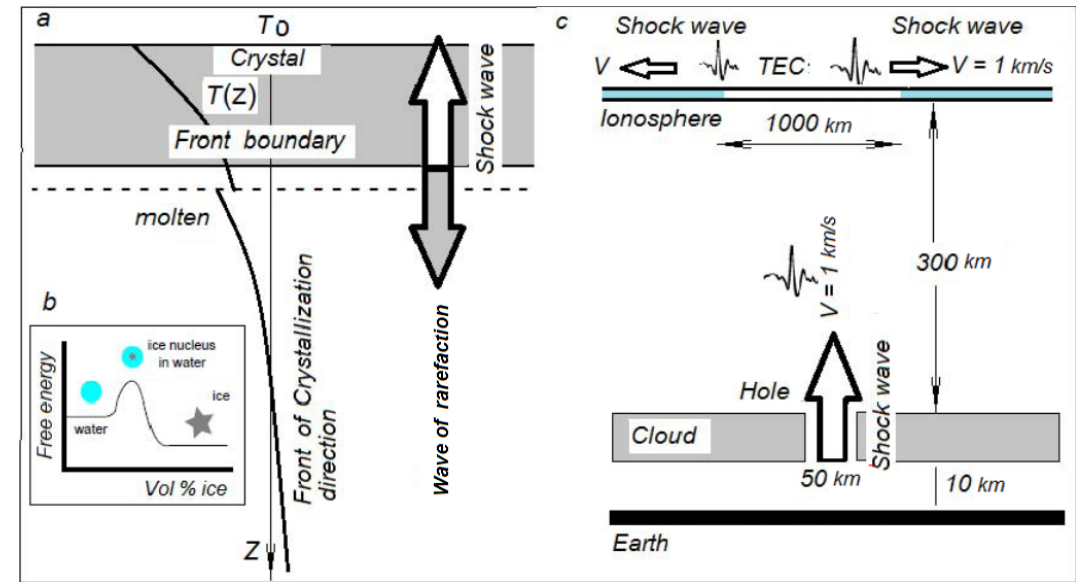

Fig. 3. The occurrence of crystallization front, rarefaction waves and SAW in supercooled water of clouds - a. Comparative particle energy (Free energy) of snowflakes, water droplets with the ice nuclei and iceless water droplets - b. Reaching ionosphere SAW is propagating through it. The hole in the cloud after the shock crystallization - $\mathrm{c}$. Note that the formation of holes in atmosphere is a well studied phenomenon that is not explained until now.

\section{Conclusion}

The article considers one of the examples of natural phenomena, which Einstein once called "spooky action at a distance". In the Earth and solar-terrestrial physics almost all phenomena in varying degrees fall under this definition. However, in physics and in mathematics the approaches to solving problems in many-particle quantum entanglement and methods for their solution are successfully being developed.

\section{References}

1. V.V. Kuznetsov Physical Mesomec. 12 (in Russian) (2009)

2. E.L. Afraimovich, N.P. Perevalova GPS-monitoring of the Earth's upper atmosphere. Irkutsk: SC RRS SB RAMS. 480 p (in Russian) (2006)

3. R.V. Row, J. Geophys. Res. 72 (1967)

4. G.V Rudenko, A.M Uralov J. Atm. Terr. Phys. 57. (1995)

5. J.P. Bardet, C. Davis, BSSA 86 (1996)

6. J.G. Anderson, G. Yu, BSSA. 86 (1996)

7. D.R. Allan, W.G. Marshall, C.R. Pulham American Mineralogist 92 (2007)

8. N.D. Kuznetsova, V.V. Kuznetsov J. of Science Forming Direction 3. (in Russian) (2015)

9. H. Roder, T. Braun, W. Schuhmann, E.Boschi, R. Buttner, B. Zimanowski EOS. Trans. AGU. 86 (2005) 